\title{
Characterisation of porcine dermis scaffolds decellularised using a novel non-enzymatic method for biomedical applications
}

Journal of Biomaterials Applications 2015, Vol. 30(2) 239-253 (C) The Author(s) 2015 Reprints and permissions: sagepub.co.uk/journalsPermissions.nav DOI: $10.1177 / 0885328215578638$ jba.sagepub.com

(S)AGE

\author{
KV Greco', L Francis', M Somasundaram ',2, G Greco', \\ Nicholas R English ${ }^{3}$, Judith A Roether ${ }^{4}$, Aldo R Boccaccini, \\ P Sibbons' ${ }^{\prime}$ and T Ansari ${ }^{I}$
}

\begin{abstract}
Off-the-shelf availability of tissue-engineered skin constructs, tailored by different combinations of reagents to produce a highly preserved biological matrix is often the only means to help patients suffering skin damage. This study assessed the effect of five different decellularisation methods on porcine dermal scaffolds with regard to matrix composition, biomechanical strength, and cytotoxicity using an in vitro biocompatibility assay. Results demonstrated that four out of the five tested decellularisation protocols were efficient in producing acellular scaffolds. Nevertheless, decellularisation method using osmotic shock without enzymatic digestion showed to be efficient not only in removing cellular material and debris from dermal scaffolds but was also beneficial in the preservation of extracellular matrix components (glycosaminoglycans and collagen). Histological assessment revealed that the dermal architecture of coarse collagen bundles was preserved. Examinations by scanning electron microscopy and transmission electron microscopy showed that the arrangement and ultrastructure of collagen fibrils in the scaffolds were retained following non-enzymatic method of decellularisation and also after collagen crosslinking using genipin. Moreover, this decellularised scaffold was not only shown to be biologically compatible when co-cultured with bone marrow-derived mesenchymal stem cells and fibroblasts, but also stimulated the cells to release trophic factors essential for tissue regeneration.
\end{abstract}

\section{Keywords}

Scaffold, dermis, fibroblast, mesenchymal stem cell, in vitro biocompatibility, genipin

\section{Introduction}

Biologic dermal scaffolds are increasingly being used to promote the regenerative process in many reconstructive surgical procedures for over a decade. ${ }^{1-4}$ The challenge facing tissue engineering, however, is to combine different reagents to preserve the structure and function of a complex mixture of proteins that forms the extracellular matrix $(\mathrm{ECM}){ }^{5}$ The ECM is highly conserved among many species and consists of molecules such as collagen, fibronectin, laminin, vitronectin, glycosaminoglycans (GAG), and growth factors oriented in a specific three-dimensional structure and composition optimised for each tissue of origin. ${ }^{6}$ Collagen is the primary mechanostructural element for dermis, conferring tensile strength and proteolytic resistance to the tissue. ${ }^{7}$ Although natural crosslinking of collagen occurs, it is still liable to degradation by the collagenase in the organism. Hence, it is often necessary to confer structural stability and collagenase resistance to

\footnotetext{
'Department of Surgical Research, NPIMR, Harrow, UK

${ }^{2}$ Nuffield Department of Surgery, John Radcliffe Hospital Headington, University of Oxford, UK

${ }^{3}$ Antigen Presentation Research Group, Imperial College London/NPIMR, Harrow, UK

${ }^{4}$ Institute of Polymer Materials, University of Erlangen-Nuremberg, Erlangen, Germany

${ }^{5}$ Institute of Biomaterials, University of Erlangen-Nuremberg, Erlangen, Germany

Corresponding author:

KV Greco, Dept. Surgical Research, NPIMR Y3, Watford RD, Harrow HAI 3UJ, UK.

Email: k.greco@ucl.ac.uk
} 
implanted materials by the introduction of exogenous crosslinking into the molecular structure, particularly when the final utilisation of the scaffold is intended to be permanent and non-absorbable. Many crosslinkers are available and the choice of a reagent that is not detrimental to the biomechanics and biocompatibility of the ECM, such as the natural-derived flavonoid genipin $^{7,8}$ may represent a good option.

ECM molecules signal cells directly or indirectly to trigger biological events and help orchestrate a set of events in a sequence to promote proliferation and differentiation of the host derived cells. ${ }^{5}$ Although the mechanisms of ECM scaffold-mediated constructive remodelling are not fully understood, stem cell recruitment $^{9,10}$ and the release of bioactive peptides by protease-mediated ECM degradation are thought to play a role in the constructive remodelling process. ${ }^{11}$ The bioactivity of scaffolds can be altered depending on the combination of decellularisation reagents used. In addition, residual chemicals from the decellularisation process can also impact on the cell's adherence and proliferation capability. ${ }^{12}$ A variety of decellularisation methods exist to produce dermal scaffolds derived from porcine skin, ranging from osmotic shock to prolonged exposure to a combination of detergents, enzymatic solutions, or acids/bases that can last days or several weeks. ${ }^{11,13,14}$ Nevertheless, decellularisation of tissues with preservation of ECM integrity and bioactivity permitting cells recruitment and remodelling can be optimised to produce an 'off-the-shelf' product for clinical application which is both time- and cost-effective.

The scope of this study was to compare five different decellularisation protocols to assess their impact on the ECM with regards to structure and composition and the ability to signal and induce migration and adhesion of bone marrow-derived mesenchymal stem cells (BMMSC) and fibroblasts.

\section{Materials and methods}

\section{Dermis harvesting}

Fresh porcine skin was obtained from Large-White/ Landrace crossbreed pigs (being terminated from unrelated studies at NPIMR) after euthanasia in a clean environment. The skin was cleaned with soap, shaved and washed with warm water. Iodine-based solution $(10 \% \mathrm{w} / \mathrm{w}$ Cutaneous Solution - Iodinated Povidone, Videne, Garforth, UK) was applied followed by a rinse with sterile PBS. A layer of the dermis (approximately $1 \mathrm{~mm}$ thick) was harvested using a dermatome (Air dermatome, Zimmer, IN, USA). Samples were cut $(3 \mathrm{~cm} \times 3 \mathrm{~cm})$ using a mould cutter and then washed in sterile PBS (PBS, Sigma-Aldrich) with $2 \%$ antibiotic/ anti-mycotic solution (AA; Sigma-Aldrich, Dorset,
UK) five times. Samples were stored in sterile plastic bags at $-20^{\circ} \mathrm{C}$ for $24 \mathrm{~h}$ as part of the decellularisation process.

\section{Decellularisation processes}

Dermis samples were defrosted and allocated into four groups ( $\mathrm{n}=12$ per group) for the production of decellularised scaffolds. Each group of samples was subjected to a different decellularisation protocol as described in Table 1. The ratio of the various solutions was $5 \mathrm{ml}$ of solution per $\mathrm{cm}^{2}$ of dermis. A brief description of each protocol is given: Protocol 1 (P1) utilises nucleases (DNase and RNase) to cleave nucleic acid sequences as the main decellularisation component. A combination of Triton-X (Sigma-Aldrich, UK), sodium deoxycholate (SOC; Fluka, Sigma-Aldrich, UK), and a wash buffer containing Tween-20 (Sigma-Aldrich, Dorset, UK) was used to remove the cellular debris from tissue. Triton is a non-ionic detergent that has the function of removing cell residues and SOC is an ionic detergent that is especially useful for disrupting and dissociating protein interactions. ${ }^{14}$

Protocol 2 (P2) utilised CHAPS (Sigma-Aldrich, UK) with ethylenediaminetetraacetate (EDTA) as a key reagent followed by sodium dodecyl sulphate (SDS) buffer (Table 1). CHAPS buffer is a zwitterionic detergent that solubilises cell membranes and dissociates DNA from proteins. EDTA is a chelating agent that aids in cell dissociation from ECM proteins by sequestering metal ions. SDS, an anionic detergent, works also as a detergent and removes nuclei from dense tissues. ${ }^{15}$ In Protocol 3 the principle of osmotic shock with consequent cell lysis was used by making use of hypertonic and hypotonic solutions followed by multiple washing steps (Table 1). This protocol was further divided into two subgroups: with (P3) and without enzymatic digestion (P3 no Enz). In the P3 samples were incubated with nucleases (DNAse and RNAse) in a buffer containing calcium $\left(\mathrm{Ca}^{+}\right)$and magnesium $\left(\mathrm{Mg}^{+}\right)$ ions. $\mathrm{Ca}^{+}$and $\mathrm{Mg}^{+}$are known to be effective in DNA degradation activity of nucleases. In the subgroup P3, no Enz nuclease enzymes were omitted in order to assess if the osmotic shock alone was able to promote decellularisation, without the need for nuclear enzymes.

Porcine dermal scaffolds in the fourth group $(n=12)$ were treated as described for Protocol 4 (P4) in Table 1. A combination of reagents was employed in this protocol, such as peracetic acid (Sigma-Aldrich, Dorset, UK) a common disinfection agent that doubles as a decellularisation agent by removing residual nucleic acids with minimal effect on the ECM composition ${ }^{14}$ and Tri(n-butyl) phosphate (Fluka, Sigma-Aldrich, Dorset, UK) an organic solvent with virucidal properties, usually used for decellularisation of dense tissues. 
Table I. Reagents, concentrations, and time used for each decellularisation protocol.

\begin{tabular}{|c|c|c|c|}
\hline Protocol I & Protocol 2 & Protocol 3 and P3 no Enz ${ }^{\mathrm{a}}$ & Protocol 4 \\
\hline Freezer $\left(-20^{\circ} \mathrm{C}\right)(24 \mathrm{~h})$ & Freezer $\left(-20^{\circ} \mathrm{C}\right)(24 \mathrm{~h})$ & Freezer $\left(-20^{\circ} \mathrm{C}\right)(24 \mathrm{~h})$ & Freezer $\left(-20^{\circ} \mathrm{C}\right)(24 \mathrm{~h})$ \\
\hline Wash buffer ${ }^{b}(12 / 12 h)$ & Wash buffer (12/I2h) & Distilled water (6h) & $\begin{array}{l}\text { Aqueous solution of } 0.1 \%(\mathrm{v} / \mathrm{v}) \\
\text { PAA and } 4 \%(\mathrm{v} / \mathrm{v}) \text { ethanol }(16 \mathrm{~h})\end{array}$ \\
\hline $\begin{array}{l}0.25 \% \text { TritonX-I00 }(\mathrm{v} / \\
\mathrm{v})+0.25 \% \text { SOC }(\mathrm{w} / \mathrm{v}) \text { in } \\
\text { PBS }+0.2 \% \text { SA }(24 \mathrm{~h})\end{array}$ & $\begin{array}{l}8 \mathrm{mM} \text { CHAPS, I M NaCl, } \\
25 \text { mM EDTA, } 0.2 \% \mathrm{SA} \\
\text { (three days) }\end{array}$ & Hypertonic solution ${ }^{c}(12 \mathrm{~h})$ & $\begin{array}{l}\text { Wash buffer (I5 } \min \times 3) \text {, followed by } \\
\text { distilled water (15 } \min \times 3)\end{array}$ \\
\hline Wash buffer (12/I2h) & $\begin{array}{l}\text { SDS buffer }{ }^{d}+0.2 \% \text { SA; } \\
\qquad \mathrm{pH}=7.4(24 \mathrm{~h})\end{array}$ & Wash buffer (8h) & $\begin{array}{l}\text { Tris buffer solution }{ }^{e} \text { with I\% (v/v) } \\
\text { Triton X-100 (90 min) }\end{array}$ \\
\hline $\begin{array}{l}\text { Incubation buffer }{ }^{\mathrm{f}}+\text { DNAse/ } \\
\text { RNAse in PBS }(24 \mathrm{~h})\end{array}$ & Wash buffer $(24 / 24 h)$ & Hypotonic solution ${ }^{\mathrm{g}}$ (12 h) & $\begin{array}{l}\text { Tris buffer solution with I\% (v/v) } \\
\text { Triton X-I00 and I\% (v/v) TnBP } \\
(90 \mathrm{~min})\end{array}$ \\
\hline Wash buffer (12/I2h) & $\begin{array}{l}\text { Dilution buffer }{ }^{h}+0.2 \% \\
\qquad S A+(48 h)\end{array}$ & Wash buffer (8h) & Wash buffer (I5 minx3) \\
\hline \multirow[t]{5}{*}{$\begin{array}{l}\text { Histological and molecular } \\
\text { analysis }\end{array}$} & Wash buffer (I5 min; 3×) & $\begin{array}{l}\text { Incubation buffer + DNAse/ } \\
\text { RNAse in PBS }(24 \mathrm{~h})\end{array}$ & $\begin{array}{l}\text { Tris buffer solution with } 2 \% \text { TnBP } \\
\text { (90 min) }\end{array}$ \\
\hline & $\begin{array}{l}\text { Histological and molecular } \\
\text { analysis }\end{array}$ & Wash buffer (12 h) & $\begin{array}{l}\text { Tris buffer solution with I\% TnBP } \\
(90 \mathrm{~min}), \text { followed by wash buffer } \\
(15 \mathrm{~min} \times 3)\end{array}$ \\
\hline & & $\begin{array}{l}\text { Histological and molecular } \\
\text { analysis }\end{array}$ & $\begin{array}{l}\text { Tris buffer solution with I\% (w/v) } \\
\text { SDS ( } 90 \mathrm{~min})\end{array}$ \\
\hline & & & $\begin{array}{l}\text { Tris buffer solution with } 0.5 \%(\mathrm{w} / \mathrm{v}) \\
\text { SDS }(90 \mathrm{~min})\end{array}$ \\
\hline & & & $\begin{array}{l}\text { Distilled water }(24 \mathrm{~h}) \text { and histo- } \\
\text { logical and molecular analysis }\end{array}$ \\
\hline
\end{tabular}

AA: antibiotic antimycotic; EDTA: ethylenediaminetetraacetic acid; PAA: paracetic acid; SA: sodium azide; SDS: sodium dodecyl sulphate; SOC: sodium deoxycholate; TnBP: tri(n-butyl)phosphate.

${ }^{a}$ For Protocol 3 without enzymatic digestion (P3 no Enz): steps in grey were not applied.

bWash buffer: PBS containing 0.05\% Tween-20.

'Hypertonic solution: I M NaCl, $10 \mathrm{mM}$ EDTA, $50 \mathrm{mM}$ Tris-HCl.

${ }^{d}$ SDS buffer: $1.8 \mathrm{mM}$ SDS, I M NaCl, and $25 \mathrm{mM}$ EDTA in I $\times$ PBS.

${ }^{\mathrm{e}}$ Tris buffer solution: Tris Base $10 \mathrm{mM}, 5 \mathrm{mM}$ EDTA, $0.4 \mathrm{mM}$ PMSF (phenylmethylsulfonylfluoride) in I $\mathrm{ml}$ EDTA, warm gently to dissolve and $0.2 \%$ sodium azide, $\mathrm{pH} 8.0$.

Incubation buffer: $400 \mathrm{mM}$ Tris- $\mathrm{HCl}, 100 \mathrm{mM} \mathrm{NaCl}, 60 \mathrm{mM} \mathrm{MgCl}$, $10 \mathrm{mM} \mathrm{CaCl}$; $\mathrm{pH} 7.6$.

gHypotonic solution: $5 \mathrm{mM}$ EDTA, $10 \mathrm{mM}$ Tris- $\mathrm{HCl}$.

hDilution buffer: I $\times$ PBS containing $10 \% \mathrm{FBS}+0.05 \%$, I\% AA.

The decellularisation processes were carried out under agitation (Incubator shaker, SciQuip, Shropshire, UK) with $1 \%$ AA (Sigma-Aldrich, Dorset, UK) added to all solutions prior to incubation to minimise bio-burden. At the end of each protocol, samples were preserved in PBS buffer (PBS, Sigma-Aldrich, Dorset, UK) prior to either molecular analysis (DNA, collagen and GAGs content) or fixation with $10 \%$ neutral buffered formalin solution $(\mathrm{NBF})$ for histological analysis.

\section{Assessment of cellular content}

Histological analysis. Samples from each protocol and a control sample were fixed for $24 \mathrm{~h}$ in $10 \%$ NBF solution at room temperature. Each sample was processed for paraffin embedding using routine laboratory techniques. Five micrometre sections were cut and stained with Haematoxylin and Eosin (H\&E) to evaluate the presence of cells and with Picro-sirius red (PSR) with Milers elastin to assess both the collagen, and elastin content and structural integrity (the latter was performed under polarised light).

DNA quantification and gel electrophoresis. To assess for the presence of DNA, dermal scaffolds were cut into small pieces, weighed, and aseptically kept in DNA-free tubes. DNA was extracted following manufacturer's instructions of GenElute mammalian genomic DNA miniprep kit (Sigma-Aldrich, UK). Total DNA was quantitated by measuring the absorbance in a nanodrop spectrophotometer (NanoDrop ND1000, Thermo Scientific, Wilmington, USA), and the absolute amount of DNA per milligram of tissue was calculated. The size, quality, and purity of the extracted DNA were 
determined by agarose gel electrophoresis. A 1.2\% agarose (Agarose Type I, low EEO; Sigma-Aldrich, Dorset, UK) gel with $1 \times$ Tris-borate-ethylenediaminetetraacetic acid (TBE - Bio Reagent, 10×, SigmaAldrich, UK) running buffer was run at $4-5 \mathrm{~V} / \mathrm{cm}$ between the electrodes. Equal volumes of DNA $(5 \mu \mathrm{l})$ and $1 \mu \mathrm{l}$ of loading buffer $(5 \times$ DNA loading buffer, Yorkshire Bioscience Ltd, York, UK) were loaded into each well. Visualisation was achieved by staining with $1 \%$ of ethidium bromide and DNA was measured via ultraviolet transillumination against a $1-\mathrm{kb}$ DNA ladder (Q-Step 4 quantitative DNA ladder, Yorkshire Bioscience Ltd, York, UK). Protocols resulting in insufficient decellularisation as assessed by histology and DNA quantification were eliminated from any further analysis.

\section{Molecular analysis of matrix components}

GAG quantification. To quantify GAG content in both the control and decellularised dermis the Blyscan GAG assay kit (Biocolor, Carrickfergus, Northern Ireland) was used. In brief, $50 \mathrm{mg}$ of finely cut wet tissue was placed in a micro-centrifuge tube and incubated with $1 \mathrm{ml}$ of papain digestion buffer at $65^{\circ} \mathrm{C}$ for $18 \mathrm{~h}$. Aliquots of each sample were mixed with 1,9dimethyl-methylene blue dye and reagents from the GAG assay kit and $200 \mu \mathrm{l}$ of each sample were added in triplicate in a 96-well plate. The absorbance was measured using a plate reader (Versamax, Molecular Devices LLC, USA) at $656 \mathrm{~nm}$ and the absolute GAG content was calculated by milligram of tissue.

Collagen assay and extraction. Collagen was extracted from decellularised dermis scaffolds using a method developed by Francis et al. (2014) (data not published). Briefly, samples were digested using a solution of guanidine hydrochloride buffer (4 M) (Sigma-Aldrich, Poole, Dorset, UK) with $12 \mu$ of protease inhibitors (SigmaAldrich, Poole, Dorset, UK) and left for $18 \mathrm{~h}$ at $4^{\circ} \mathrm{C}$ under agitation. Samples were then centrifuged for $10 \mathrm{~min}$ at $12,000 \mathrm{~g}$ to separate tissue debris. Supernatant was transferred to a micro-centrifuge tube and nuclear material was digested by using RNAse enzyme (Sigma-Aldrich, Dorset, UK; activity of $140 \mathrm{EU} / \mathrm{ml}$ ) for $2 \mathrm{~min}$ at RT, followed by DNAse I (Sigma-Aldrich, Dorset, UK; activity of $100 \mathrm{EU} / \mathrm{ml}$ ). Samples were agitated without vortexing and incubated at $37^{\circ} \mathrm{C}$ for $1 \mathrm{~h}$, and then transferred to a dialyser with a NMWCO (3000 Da) and dialysed against distilled water overnight at $4^{\circ} \mathrm{C}$. After dialysis supernatant was transferred to an amicon ultra filter 2 (Ultra-4 $3 \mathrm{kDa}$ Ultracel-PL memb, Merk Millipore, Bilerica, MA, USA) to remove the hydrolysed DNA and salts, and then centrifuged for $30 \mathrm{~min}$ at $4000 \mathrm{~g}$. For protein isolation, $50 \mu \mathrm{l}$ of Tris base ( $\mathrm{pH} 9.5)$ followed by icecold phenol-chloroform-isoamyl alcohol (PCIA, Sigma- Aldrich, Dorset, UK) was added to the retenate, which was allowed to stand for $24 \mathrm{~h}$ at $4{ }^{\circ} \mathrm{C}$, and then centrifuged for $10 \mathrm{~min}$ at $12,000 \mathrm{~g}$. The upper layer (containing DNA/RNA) and the lower organic phase (containing solvents) were removed by pipetting. The interface containing proteins was carefully removed and transferred to another micro-centrifuge tube. Protein was precipitated using ice-cold ethanol and centrifuged for $10 \mathrm{~min}$ at $15,000 \mathrm{~g}$ at $4^{\circ} \mathrm{C}$. Pellet was allowed to dry and solubilised with $100 \mu$ l of ultrapure acidic water $(\mathrm{pH}=3$ with acetic acid).

Collagen quantification. Collagen was quantified using Sircol collagen assay kit (Biocolor, Carrickfergus, Northern Ireland). Briefly, $90 \mu \mathrm{l}$ of each sample was subjected to the Sirius red dye binding, followed by wash with ice-cold acid-salt reagent to remove unbound dye. Samples were centrifuged and the dye bound to collagen was released from the pellet with alkali reagent $(1 \mathrm{~N} \mathrm{NaOH})$. Extracts were placed in a 96-well plate in triplicates and spectrophotometric readings were taken at $555 \mathrm{~nm}$ on a microplate reader (Versamax, Molecular Devices LLC, USA). Absolute values were attained with a standard curve composed of type I bovine skin collagen solution $(0.5 \mathrm{mg} / \mathrm{ml})$ in the range of $5-100 \mu \mathrm{g}$ per $0.1 \mathrm{ml}$. Total collagen was normalised per milligram of tissue.

Sodium dodecyl sulfate-polyacrylamide gel electrophores (SDS-PAGE). Ten microliters of each sample was taken and separated using gradient pre-cast $4-15 \%$ polyacrylamide gels of $1 \mathrm{~mm}$ thickness (Mini-PROTEAN TGX Stain-Free Precast Gel, Bio-Rad Laboratories Inc., USA) and run with the gel system apparatus (MiniPROTEAN Tetra System, Bio-Rad Laboratories Inc., USA) at $150 \mathrm{~V}$ for $2 \mathrm{~h}$. Protein bands on gel were stained with coomassie blue (Coomassie blue R350, Invitrogen, UK) and allowed to de-stain in water for 24-28 h. Images were taken to qualitatively assess the protein fractions compared to control samples.

Crosslinking of the decellularised scaffolds. Decellularised dermis samples from protocols P1, P3, and P3 no Enz $(n=4$ in each group) were crosslinked with genipin ( 0.5 and $1 \% \mathrm{w} / \mathrm{v}$ in water) (Wako Chemicals USA, Richmond, USA) for $5 \mathrm{~h}$, under agitation, at room temperature. The ratio of volume of genipin and scaffold was $0.5 \mathrm{ml} / \mathrm{cm}^{2}$. The crosslinked tissues were then thoroughly washed (four washes of $10 \mathrm{~min}$ ) with sterile PBS containing $1 \%$ AA solution and stored at $4{ }^{\circ} \mathrm{C}$ prior to mechanical testing. Control samples were kept in sterile PBS containing 1\% AA solution under the same conditions for comparison. 


\section{Biomechanical tests}

Mechanical properties of control, decellularised and genipin-treated dermal scaffolds ( $\mathrm{n}=4$ for each group) were evaluated by tensile strength test measured as the maximum stress that each sample could withstand while being stretched before reaching breaking point. For each test, specimens were cut into 'dumbbell' shapes using a standard mould with a length of $2.5 \mathrm{~cm}$ and width of $0.4 \mathrm{~mm}$. The tests were performed with the application of uniaxial tension using a tensiometer (Instron Inspec 220 Benchtop Portable tester, Instron, Buckinghamshire, UK). Samples were clamped into the holders and loaded at a constant tension rate. The tensile tester recorded the real-time load and elongation to which the tissue was subjected. Parameters such as maximum load $(\mathrm{N})$, testing time (s), and extension at maximum load $(\mathrm{mm})$ were recorded. Strain was defined as rate of change of sample deformation and calculated as ratio of the length at the maximum load to the original length, and stress defined as how much load was applied at each meter squared sample. Young's modulus (YM; MPa) was calculated by the ratio of stress $\left(\mathrm{N} / \mathrm{m}^{2}\right)$ over strain $(\%)$.

\section{Ultrastructural assessment of scaffolds}

The morphological profiles of decellularised dermis samples with and without crosslinking (genipin $0.5 \% ; \mathrm{w} / \mathrm{v}$ ) were observed under scanning electron microscopy (SEM) and transmission electron microscopy (TEM). For SEM analysis, samples were fixed with $3 \%(\mathrm{v} / \mathrm{v})$ glutaraldehyde (Sigma-Aldrich, Dorset, UK) $0.1 \mathrm{M}$ in phosphate buffer and freeze-dried. Samples were placed on $8 \mathrm{~mm}$ diameter aluminium stubs and sputter coated. Collagen morphology was examined under a SEM (LEO 435VP, Zeiss Leica). Samples were scanned and the micrographs were recorded. For TEM observation, rehydrated dermal scaffolds and control samples were fixed in 3\% glutaraldehyde in $0.1 \mathrm{M}$ sodium phosphate buffer $\mathrm{pH} 7.4$ at room temperature and post-fixed in $1 \%$ osmium tetroxide (Agar Scientific, Stansted UK) in PBS pH 7.4 for $1 \mathrm{~h}$. After thorough washing, the samples were block stained in 2\% Uranyl acetate (Agar Scientific, Stansted, UK) for 2-4h. Samples were then washed with distilled water, dehydrated using an acetone gradient (BDH Merck Ltd, Poole, Dorset, UK), and gradually infiltrated with araldite resin (Agar Scientific, Stansted UK) in a ratio of 1:1 (acetone:araldite). After infiltration with araldite resin for up to $8 \mathrm{~h}$, the samples were embedded and cured for at least $18 \mathrm{~h}$ at $65^{\circ} \mathrm{C}$. The ultrathin sections $(100 \mathrm{~nm}$ thick; using a Reichert-Jung Ultracut E microtome) were collected on 200 mesh copper grids (Agar Scientific, Stansted, UK). They were stained with Reynold's Lead citrate and carbon coated and viewed using a Jeol JEM1200 EX electron microscope (Tokyo, Japan) to investigate particles' ultrastructure.

\section{In vitro biocompatibility assessment}

\section{Cells isolation and culture}

BM-MSC. Bone marrow was aspirated from Landrace crossbred pigs. All the animal procedures complied with institutional ethical use protocols (such as stated in the NIH Guide for Care and Use of Laboratory Animals). Under anaesthesia and sterile conditions, the hind limb was extended and a $1 \mathrm{~cm}$ transverse cutaneous incision was made medially at the mid-point of the femur. Bone marrow aspiration was performed using a heparinised $11 \mathrm{G}$ Jamshidi needle. Aspiration yield varied from between 20 and $60 \mathrm{ml}$ based on age and pre-morbid state of the animal. Bone marrow aspirates underwent gradient separation using Histopaque ${ }^{\circledR}$ (Sigma Aldrich, UK) at a 1:1 ratio. Tubes were centrifuged at $400 \mathrm{~g}$ for $40 \mathrm{~min}$ and cellular monolayer carefully extracted before washing in $10 \mathrm{ml}$ PBS at $250 \mathrm{~g}$ for $10 \mathrm{~min}$. A further two washes in PBS were performed. Pellets were reconstituted in $10 \mathrm{ml}$ of Alpha Minimum Essential Medium ( $\alpha$ MEM - Sigma Aldrich, UK) and plated in T75 flasks. Incubation took place at $37.5^{\circ} \mathrm{C}$ and $5 \% \mathrm{CO}_{2}$ in $\alpha$ MEM, $10 \%$ foetal bovine serum (FBS; Hyclone/ ThermoScientific, Rockford, MD, USA) and $1 \%$ of a AA solution (Sigma-Aldrich, Dorset, UK) (growth medium). Flasks were reviewed daily and washed after $48 \mathrm{~h}$ to remove unwanted cells and debris. Medium was changed every $48 \mathrm{~h}$. BM-MSC reached confluence after approximately five days and were split at ratio 1:2 using 0.25\% Trypsin-EDTA (Sigma Aldrich, UK) up to passage 3. The immunophenotype of BM-MSC was tested by flow cytometry and was negative for the leukocyte markers CD34 and CD14 and positive for the stem cell markers CD44, CD90, CD29 (data not shown).

Fibroblasts. Fibroblasts were isolated from dermis from the same pigs. Dermis was removed as previously described. Dermal layers were cut into $1 \mathrm{~cm} \times 1 \mathrm{~cm}$ pieces, washed 10 times with $2 \%$ AA in PBS and placed in a Petri dish. DMEM (Sigma-Aldrich, Dorset, UK) with $10 \%$ FBS (Hyclone/ThermoScientific, Rockford, MD, USA) and 1\% AA (Sigma-Aldrich, Dorset, UK) was added to the tissue explants, which were left undisturbed for one week in a humidified incubator $\left(37^{\circ} \mathrm{C}, 5 \% \mathrm{CO}_{2}\right)$ to allow the outgrowth of fibroblasts. Prior to the assays, both BM-MSC and fibroblasts were released from the flasks using a cell dissociation solution (Cell Dissociation Solution Nonenzymatic $1 \times$ Sigma-Aldrich, Dorset, UK). Both cells were utilised at passage 2 for the in vitro assays.

In vitro co-culture system. A modified in vitro Boyden-type chemotaxis assay was developed to test 
cytotoxicity of scaffolds, its capacity to signal cell migration through a $12 \mu \mathrm{m}$ pore size membrane of transwell inserts (Millicell-PCF, Millipore, Billerica MA, USA), and adherence on acellular scaffolds. Scaffolds were incubated in Dulbecco's Phosphate Buffered Saline $1 \times$ with $\mathrm{Ca}^{2+}$ and $\mathrm{Mg}^{2+}$ (DPBS; Invitrogen, UK) for $24 \mathrm{~h}$ and then in growth medium overnight at $37.5^{\circ} \mathrm{C}$ and $5 \% \mathrm{CO}_{2}$ previously to the in vitro assay. BM-MSC and scaffold were co-cultured in different compartments (insert and well, respectively) permitting physical separation but communication via paracrine signalling in the system (Figure 4(a)). Prior to the in vitro assay, genipin-treated and decellularised dermis arising from protocols P3 or P3 no Enz were cut into $0.5 \mathrm{~cm} \times 0.5 \mathrm{~cm}$ incubated in DPBS containing $\mathrm{Ca}^{2+}$ and $\mathrm{Mg}^{2+}$ (DPBX $1 \times$, Invitrogen, UK) for $24 \mathrm{~h}$ and then in growth medium overnight at $37.5^{\circ} \mathrm{C}$ and $5 \% \mathrm{CO}_{2}$. Specimens were placed in a 24 -well plate and $\mathrm{BM}$-MSC cells were plated in the inserts at the density of $5 \times 10^{4}$ cells $/ \mathrm{cm}^{2}$. The same concentration of both cells was seeded in empty polystyrene distinct wells as control. After 48 and $72 \mathrm{~h}$, scaffolds were collected, gently washed with PBS fixed in $10 \%$ NBF for $24 \mathrm{~h}$. Media was changed every $24 \mathrm{~h}$ and samples from each time point were frozen at $-80^{\circ} \mathrm{C}$ for ELISA analysis. Following fixation, scaffolds were embedded in 3\% agar (Select Agar, Invitrogen, UK) in distilled water (w/v) and processed for subsequent histological assessment.

Proliferation assay. Scaffolds were prepared as described in Materials and Methods prior to this assay. Porcine primary fibroblasts were seeded on each dermis sample in triplicate, at $2.5 \times 10^{5}$ cells $/ \mathrm{cm}^{2}$ of scaffolds. Cells were allowed to adhere for $2 \mathrm{~h}$ in the incubator $\left(37^{\circ} \mathrm{C}\right.$ and $\left.5 \% \mathrm{CO}_{2}\right)$ and then fresh medium (DMEM, 10\% FBS and 1\% AA) was added into each well. Medium was changed every $24 \mathrm{~h}$ and frozen at $-80^{\circ} \mathrm{C}$ for subsequent analysis. After 48 and $72 \mathrm{~h}$, scaffolds were collected, gently washed with PBS, and tested for DNA content as described previously. DNA starting amount was measured after cells attachment at day 0 and used to normalise following time points (48 and $72 \mathrm{~h}$ ). Data were expressed as fold change compared to day 0 .

Growth factor and cytokines assay. Production of growth factor and cytokines in the supernatant by BM-MSC and fibroblasts when cultured with dermal scaffolds was measured as another read-out of cytotoxicity and phenotype differentiation. Concentrations of porcine TGF- $\beta 1$ (R\&D Systems, Minneapolis, MN), IL-10 (Invitrogen, Frederick, MD, USA), and Tumour Necrosis Factor-alpha (TNFa) (Invitrogen, Frederick, MD, USA) were measured in the culture medium by ELISA. Manufacturer's instructions were followed for all three assays. Supernatant of cells (BM-MSC or fibroblasts) cultured without scaffolds were used as controls. Each assay was performed in triplicate and from three distinct experiments.

\section{Statistical analysis}

Data were calculated as mean \pm standard error, and significance was determined by performing two-tailed Student's $t$-tests or ANOVA (Prism 5: Graphpad Software, La Jolla, California), followed by Bonferroni's post hoc test. A $p$ value of less than 0.05 was considered to be significant.

\section{Results}

\section{Decellularisation protocols for porcine dermis}

Different decellularisation protocols were applied to porcine dermis, each protocol is presented in detail in Table 1. Following decellularisation, samples were assessed histologically using H\&E-stained sections. Figure 1(a) shows the histological structure of pig dermis from each protocol together with a control sample. The control H\&E-stained sample shows the collagen layer in pink, which corresponds to the reticular layer of dermis with collagen fibres arranged in parallel to the surface of the skin. The nuclei of the cells (as pointed by arrows) were stained purple/blue. The PSRstained sections showed the classical collagen fibre architecture, which when viewed under polarised light due to birefringence appears red-orange in colour. Images of $\mathrm{H} \& \mathrm{E}$-stained decellularised dermis using protocols 1, 2, 3, and 3 with no enzymatic digestion (P3 no Enz) revealed the complete removal of cells and nuclear material from the dermal scaffolds (Figure 1(a)). However, nuclei were still evident in the tissue when protocol 4 was used to decellularise the dermis. Furthermore, DNA quantification showed that approximately $90 \%$ of nuclear material was depleted by all decellularisation processes (Table 2) when compared to control dermis, with no visible DNA on the electrophoresis gel following staining by ethidium bromide (Figure 1(c)). The exception was again P4 whose quantification $(121.81 \pm 4.12 \mathrm{ng}$ $\mathrm{DNA} / \mathrm{mg}$ tissue) showed no difference in comparison to the control sample $(289.93 \pm 4.6 \mathrm{ng} \mathrm{DNA} / \mathrm{mg}$ tissue $)$ (Table 2). PSR-stained polarised light images show the arrangement of collagen fibres was preserved after all decellularisation methods. Interestingly, the elastin fibres of P3 no Enz (arrow head; stained by Miller elastin staining in dark blue) were as well preserved after and comparable only to the control dermis (Figure 1(a)). Based on these results, Protocols P4 (inadequate decellularisation) and P2 (low cost-effectiveness) were 

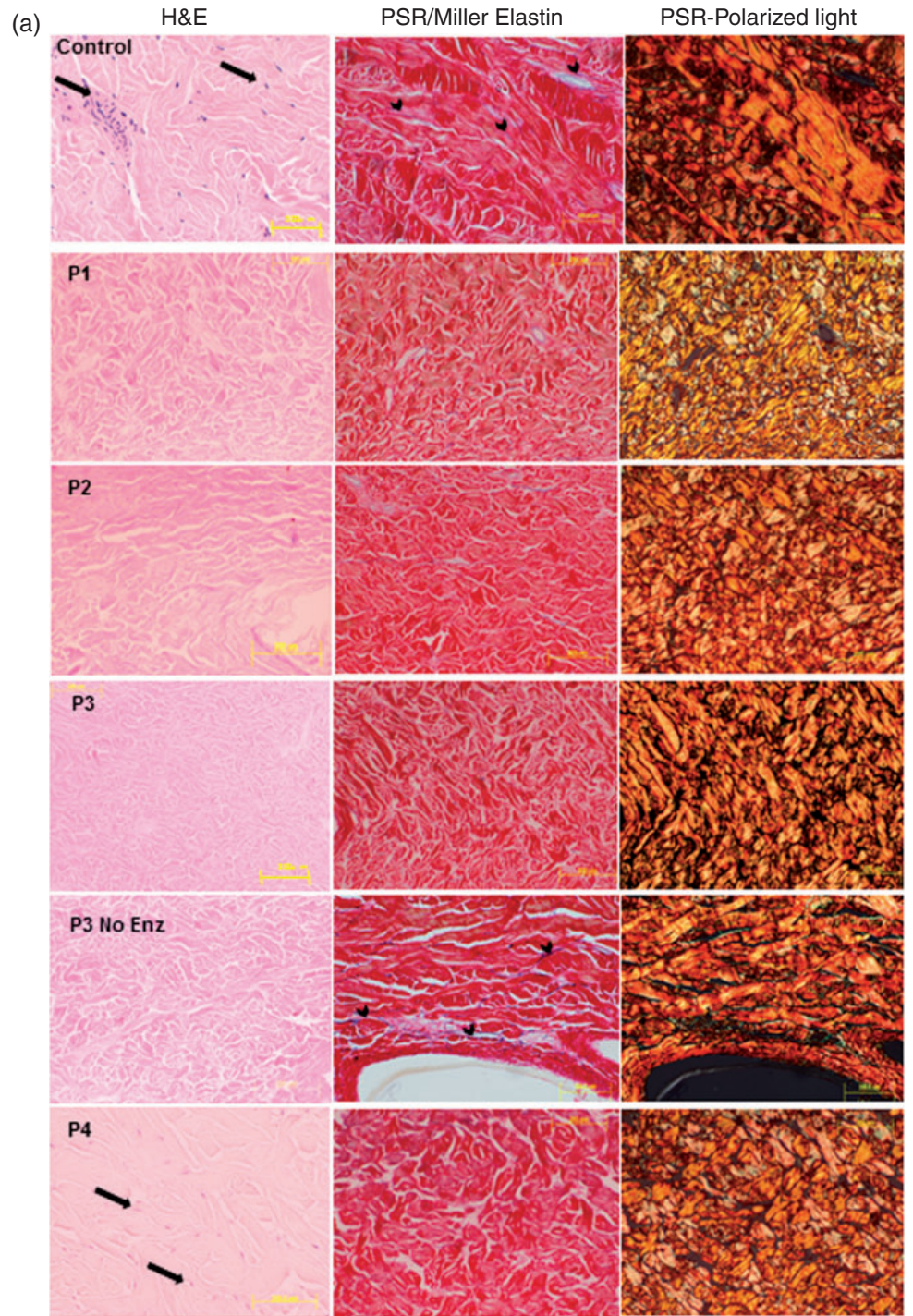

(b)

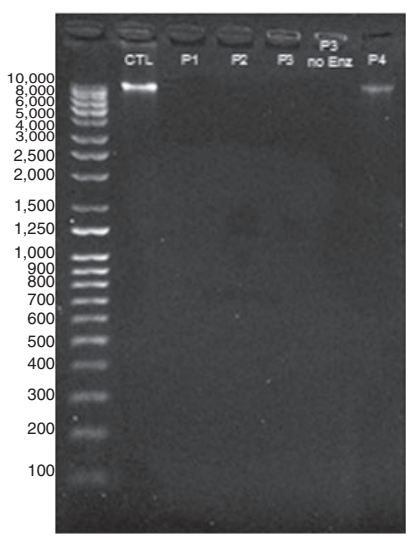

(c)

\begin{tabular}{cc}
\hline Sample & DNA(ng/mg) \\
CTL & $296.93 \pm 4.60$ \\
P1 & $36.00 \pm 3.64^{*}$ \\
P2 & $42.56 \pm 2.80^{*}$ \\
P3 & $38.92 \pm 3.84^{*}$ \\
P3 no Enz & $35.12 \pm 2.12^{*}$ \\
P4 & $121.81 \pm 4.12$ \\
\hline
\end{tabular}

Figure I. (a) Evaluation of porcine dermis scaffolds after different decellularisation protocols. Control dermis shows the presence of intact cells with nuclei (arrow) in the matrix stained with Haematoxylin and Eosin (H\&E), intact collagen fibres stained with Picro-sirius red (PSR; taken under bright field microscopic light) with presence of elastin fibres (arrow head) stained in blue with Millers elastin (ME). Collagen fibres of control tissue after PSR staining under polarised light, display red-orange colour due to birefringence. After decellularisation using protocols PI, P2, P3, and P3 with no enzymatic digestion (P3 no Enz), dermal scaffolds show no apparent presence of cells when stained with $\mathrm{H} \& \mathrm{E}$, and preserved collagen macro-architecture when stained with PSR/polarised light.

Nevertheless, the collagen fibres bundles in PI and P3 have a thinner and looser appearance. P3 no Enz also shows conserved elastin fibres stained with ME (arrow head). (b) Digital image of electrophoresis gel with six samples run on I.2\% agarose and stained with ethidium bromide. The DNA size marker is a commercial $10 \mathrm{kbp}$ ladder. The position of the wells is noted in all samples, and direction of DNA migration is noted only in sample control (native dermis) and dermal scaffold obtained from protocol 4 (P4). (c) Quantification of DNA (ng/mg) extracted from each sample. ${ }^{*} P<0.05$ significantly different from control (CTL). 
Table 2. Mechanical properties of dermal matrices: tensile strength test.

\begin{tabular}{lllll}
\hline Samples & $\begin{array}{l}\text { Genipin } \\
(\%)\end{array}$ & $\begin{array}{l}\text { Stress } \\
\left(\mathrm{N} / \mathrm{m}^{2}\right) \times 10^{6}\end{array}$ & Strain (\%) & $\mathrm{YM}(\mathrm{MPa})$ \\
\hline CTL & - & $15.85 \pm 1.12$ & $0.48 \pm 0.03$ & $33.98 \pm 2$ \\
PI & - & $11.0 \pm 0.73$ & $0.40 \pm 0.02$ & $27.89 \pm 2.5^{*}$ \\
PI & 0.5 & $14.12 \pm 0.65$ & $0.48 \pm 0.06$ & $29.37 \pm 2$ \\
PI & I & $14.57 \pm 0.8$ & $0.50 \pm 0.02$ & $29.73 \pm 1.9$ \\
P3 & - & $10.77 \pm 0.97$ & $0.41 \pm 0.02$ & $26.10 \pm 2^{*}$ \\
P3 & 0.5 & $14.44 \pm 1.27$ & $0.50 \pm 0.02$ & $29.43 \pm 1.8$ \\
P3 & I & $14.94 \pm 1.1$ & $0.49 \pm 0.03$ & $30.46 \pm 1.2$ \\
P3 no Enz & - & $14.49 \pm 0.21$ & $0.46 \pm 0.02$ & $31.39 \pm 2$ \\
P3 no Enz & 0.5 & $16.00 \pm 1.32$ & $0.46 \pm 0.01$ & $34.84 \pm 2.8$ \\
P3 no Enz & I & $15.65 \pm 2.0$ & $0.44 \pm 0.01$ & $35.09 \pm 3.8$ \\
\hline
\end{tabular}

Comparison of representative values of stress $\left(\mathrm{N} / \mathrm{cm}^{2}\right)$, strain (\%), and the Young's modulus (YM; MPa). YM (ratio of stress over strain) was taken for comparison among samples: control (CTL), and scaffolds obtained after protocols $\mathrm{P} 3$ no Enz, P3, and PI, crosslinked with genipin $(0.5$ and $1 \% \mathrm{w} / \mathrm{v})$ or non-crosslinked. $* P<0.05$ significantly different from control (CTL).

excluded from any further characterisation and analysis.

\section{Molecular analysis of matrix components}

The ECM components of the bioengineered dermal scaffolds were assessed by quantification of collagen and GAGs. The amount of GAGs (Figure 2(a)) and collagen (Figure 2(b)) extracted from the samples decreased when compared to control samples. Amongst the three decellularisation methods, although not significant, P3 no Enz showed a tendency for preserving greater amount of both GAGs $(0.23 \pm 0.02 \mu \mathrm{g}$ $\mathrm{GAG} / \mathrm{mg}$ tissue) and collagen $(7.3 \pm 1.07 \mu \mathrm{g}$ collagen/ $\mathrm{mg}$ tissue) when compared to control $(\mathrm{GAG}=0.32 \pm 0.03 \mu \mathrm{g} \quad \mathrm{GAG} / \mathrm{mg} \quad$ tissue; collagen $=9.96 \pm 1.11 \mu \mathrm{g}$ collagen $/ \mathrm{mg}$ tissue) (Figure 2(a) and (b)). Figure 2(c) shows the SDS-PAGE electrophoresis patterns of the commercial marker and four collagen samples as follows: control dermis, scaffolds obtained from P1, P3, and P3 no Enz. No change was observed in the distribution of the type I collagen bands in the decellularised samples when compared to control. However, sample P3 no Enz appears to be more similar to the control in thickness, which implies that a higher amount of collagen remained in the matrix after this decellularisation process.

\section{Crosslinking effects on the dermal scaffolds}

A SEM image of a non-decellularised porcine skin is shown in Figure 3(a). It exhibits a compact structure of
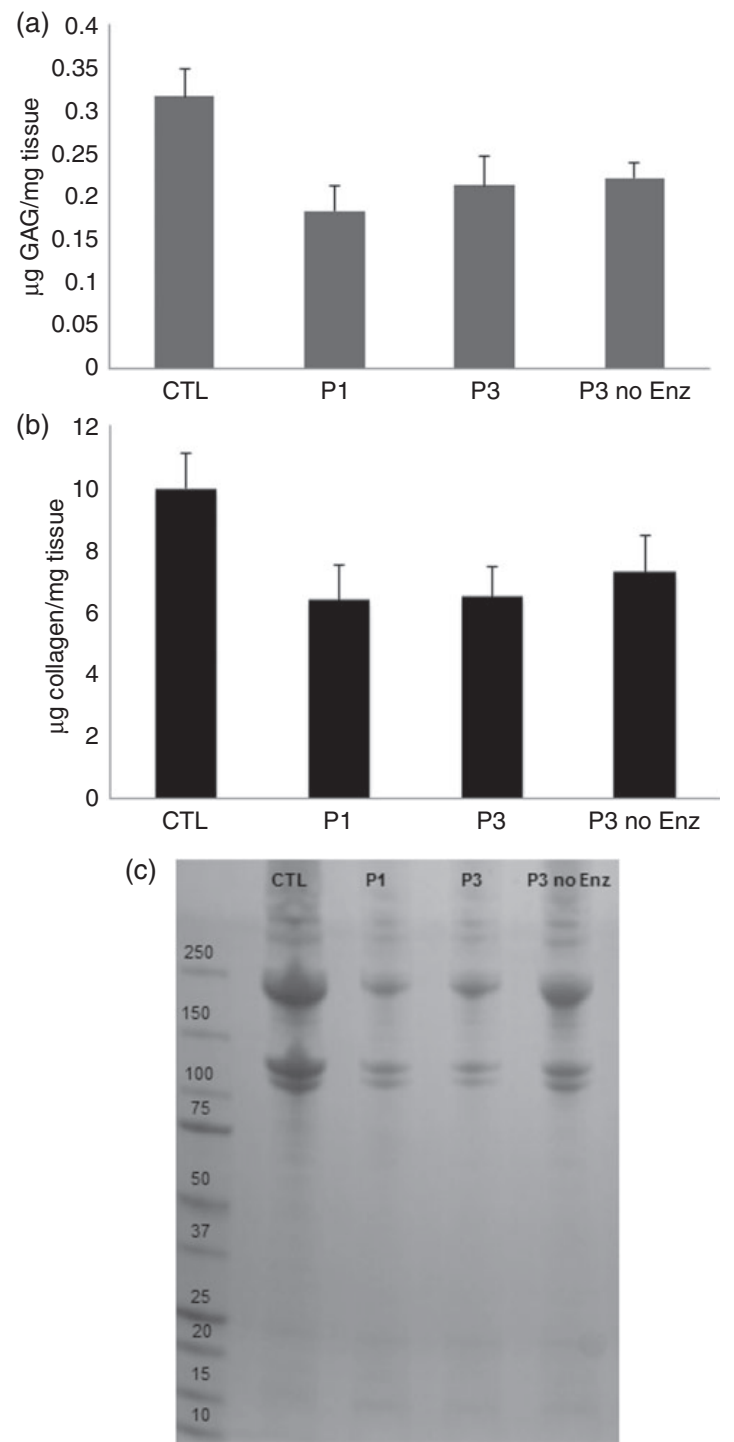

Figure 2. Characterisation of extracellular matrix. Quantitative analysis of (a) glycosaminoglycans (GAGs) and (b) collagen contents measured on the scaffolds after decellularisation protocols PI, P3, and P3 no Enz, compared to control dermis. (c) Digital image of SDS-PAGE electrophoresis gel showing the patterns of collagen type I bands (stained with coomassie blue) in the control sample, and dermal scaffolds obtained after decellularisation protocols $\mathrm{PI}, \mathrm{P} 3$, and $\mathrm{P} 3$ no Enz. The protein marker is a commercial ladder with $10-250 \mathrm{kDa}$.

collagen fibres where architecture of the collagen is shown to be dense and continuous. Samples that underwent a period of enzymatic digestion (P1 and P3) show a looser meshwork of collagen fibres (Figure 3(b) and (c), respectively) when compared to P3 no Enz (Figure 3(d)). Non-crosslinked scaffolds appear to have the appendages and basic dermal architecture of the collagen, however in a more loosened meshwork (Figure 3(b) to (d)) when compared to control (Figure 3(a)) and to the genipin crosslinked samples 

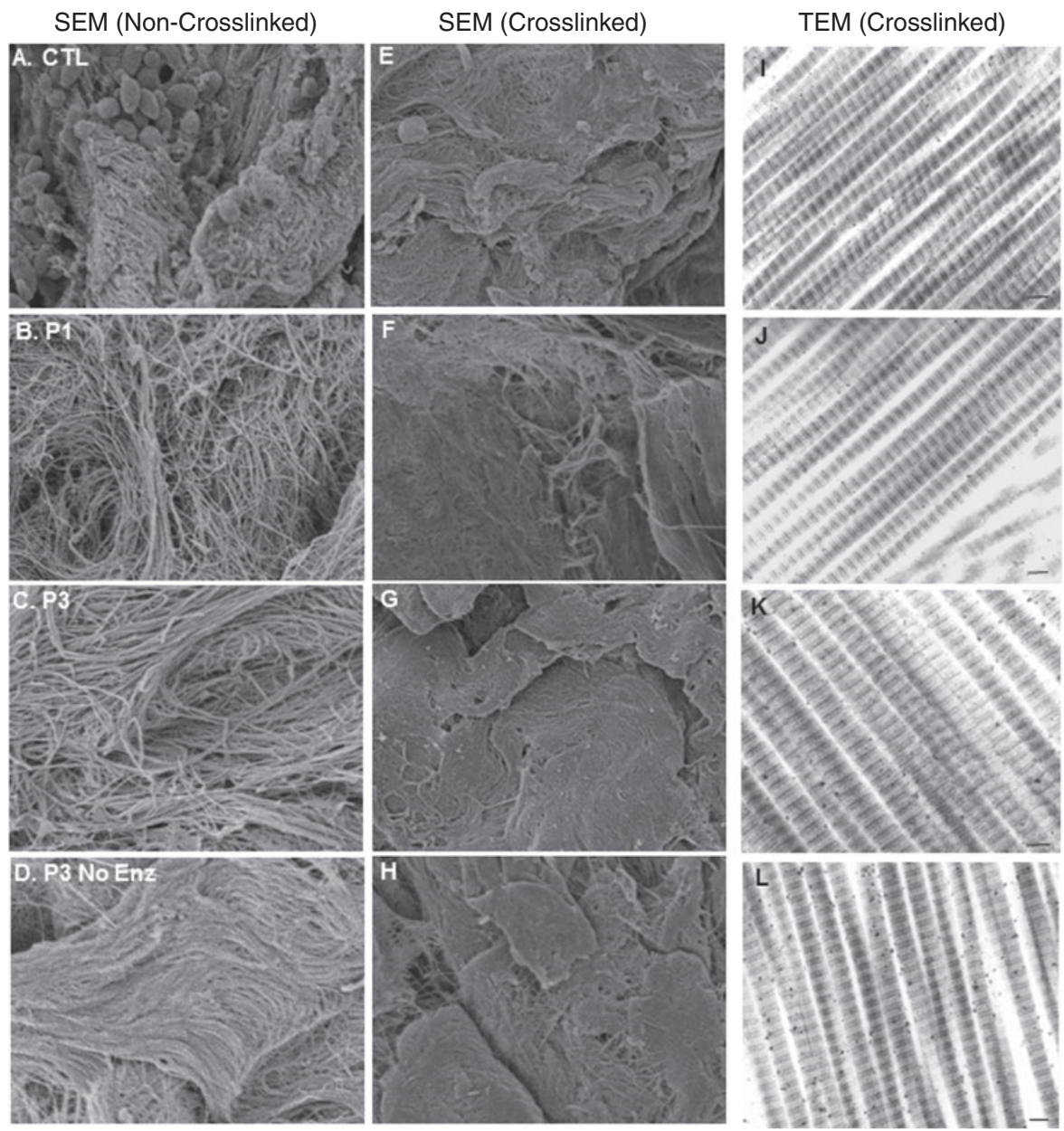

Figure 3. Ultrastructural analysis of dermal scaffolds. SEM micrographs of non-crosslinked (a) control and scaffolds obtained from (b) PI, (c) P3, and (d) P3 no Enz. (e-h) SEM images of the respective scaffolds but crosslinked with genipin 0.5\% (w/v). Magnification of SEM images: $3000 \times$. TEM images obtained from samples crosslinked with genipin (0.5\%): (i) control, (j) PI, (k) P3, and (l) P3 no Enz. Bar: $100 \mathrm{~nm}$.

(Figure 3(f) to (h)). TEM images for dermal scaffolds crosslinked with genipin (Figure 3(j) to (l)) show a sequential arrangement of fibrils in longitudinal crosssections with regular contours and orientation, comparable to those seen in the control dermis (Figure 3(1)).

\section{Mechanical properties: Tensile strength test}

The mechanical properties of scaffolds were investigated by the tensile strength test (Table 2). Crosslinking with genipin (both 0.5 and $1 \%$ ) did not substantially affect dermal scaffolds; however, a significant decrease in the YM was observed in the non-crosslinked P1 $(27.89 \pm 2.5 \mathrm{MPa})$ and $\mathrm{P} 3(26.1 \pm 2 \mathrm{MPa})$ samples when compared to control $(33.89 \pm 2 \mathrm{MPa})$. Interestingly, P3 no Enz crosslinked with both 0.5 and $1 \%$ genipin had highest YM result $(34.84 \pm 2.8$ and $35.09 \pm 3.8 \mathrm{MPa}$, respectively) (Table 2).

\section{Biocompatibility tests}

The biocompatibility of the dermal scaffolds after P3 and P3 no Enz decellularisation methods were confirmed by evaluation of a modified in vitro Boydentype chemotaxis assay (Figure 4(a)). The cytotoxicity of the scaffold, matrix degradation products, and remaining chemicals from decellularisation process were tested by paracrine signalling on cell transmigration and adhesion. Both dermal samples were effective in stimulating cell migration and adhesion. Histological examinations showed that on day 2 cell transmigration was evident, as the cells were attached on the acellular scaffolds forming a continuous layer of cells on top of the scaffolds (Figure 4(b)). After $72 \mathrm{~h}$ few migrated cells were also found in the scaffolds (arrows). In the absence of scaffolds, cells seeded on the inserts failed to transmigrate (showed as CTL in Figure 4(c)). 


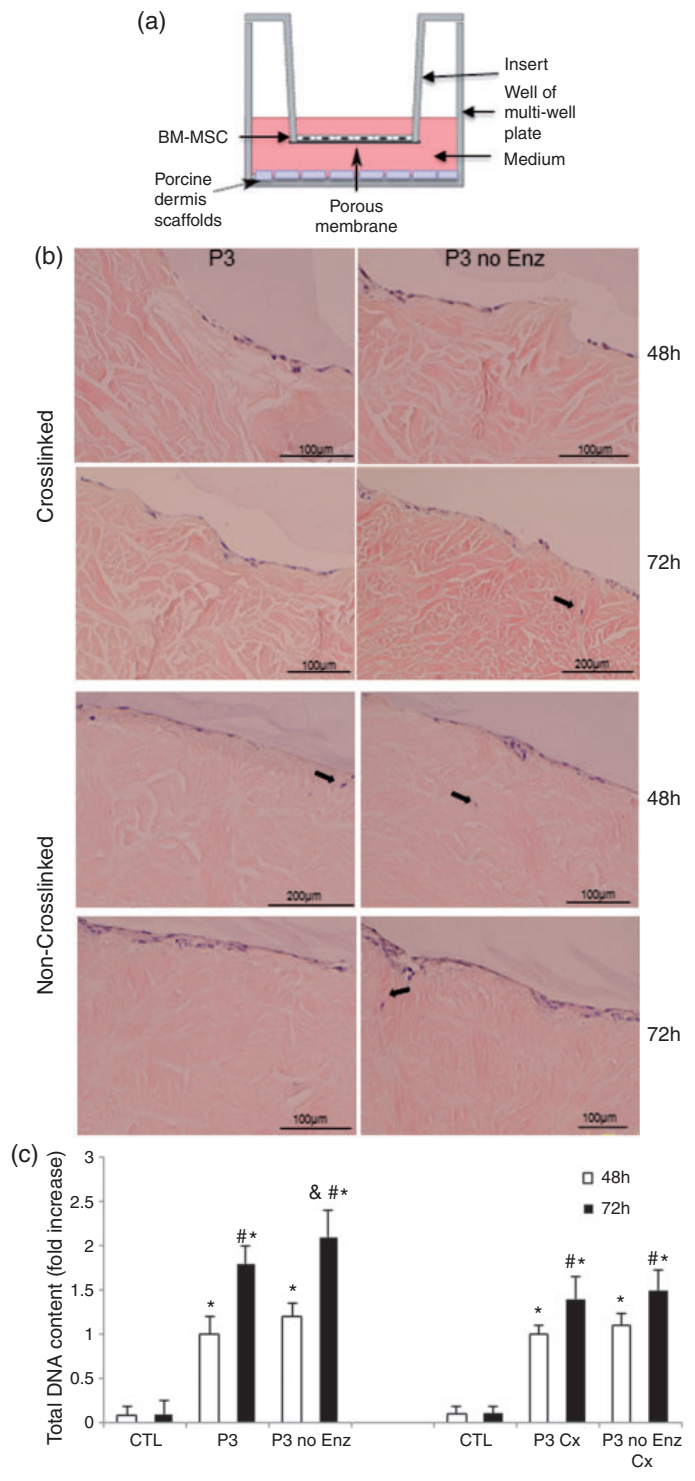

Figure 4. In vitro biocompatibility test. (a) Scheme of a modified Boyden-type chemotaxis assay: small pieces $(0.5 \mathrm{~cm} \times$ $0.5 \mathrm{~cm}$ ) of porcine dermal scaffolds (P3 or P3 no Enz), crosslinked with genipin $0.5 \%(\mathrm{Cx})$ or non-crosslinked are placed in the bottom of a well of a multi-well plate. Bone marrow-derived mesenchymal stem cells (BM-MSC) are seeded in the insert placed on the well and allowed to interact with the scaffolds via paracrine signalling and eventually migrate towards them. After 48 and $72 \mathrm{~h}$ scaffolds were collected and processed for histological analysis. (b) Digital images of the histological sections stained with H\&E show the scaffolds with a layer of BM-MSC on top and some migrated ones (arrows). (c) Cell proliferation was assessed by total DNA content after co-culture of porcine primary fibroblasts with dermal scaffolds for 48 and $72 \mathrm{~h}$.

Results are expressed as fold increase compared to day 0 . CTLs are the total DNA of the cells that have potentially migrated and adhered to the well plate in absence of the scaffold. $* P<0.005$ significantly different when compared to the CTL. $P<0.05$ significantly different when comparing the same protocol after $72 \mathrm{~h}$ of co-culture (\#) or compared to the other protocol at the same time-point (\&).
Cell proliferation was assessed by total DNA content by using porcine primary fibroblasts cultured in contact with scaffolds (Figure 4(c)). DNA quantification demonstrated that scaffolds supported continued cell growth. After $72 \mathrm{~h}$ a significant augmented cell proliferation $(>45 \%)$ was observed in the non-crosslinked scaffolds, and approximately $+25 \%$ in the crosslinked ones when compared to $48 \mathrm{~h}$ (Figure 4(c)). Samples of supernatant were collected at each time point to assess production of TGF- $\beta 1$, IL-10, and TNF- $\alpha$ (Figure 5). Results showed that BM-MSC significantly increased production of TGF- $\beta 1$ at all time points and on all scaffolds. Fibroblast, however, had a consistent production of TGF- $\beta 1$ throughout. IL-10 production for both cells was significantly enhanced when in co-culture with all scaffolds. The release of TNF- $\alpha$ in the supernatant was distinctly augmented by BM-MSC when cultured with P3 no Enz decellularised dermis after 48 and $72 \mathrm{~h}$.

\section{Discussion}

\section{Scaffolds development and characterisation}

In this study, five different methods for cell removal were tested, encompassing different combinations of enzymes and detergents in order to produce acellular dermal scaffolds. They were assessed according to their mechanical and functional properties and capacity to promote cell recruitment and proliferation using an in vitro biocompatibility assay. We have demonstrated that protocols 1 (P1), $2(\mathrm{P} 2)$, and $3(\mathrm{P} 3)$ were effective in removing nearly all cellular elements without evidence of major damage on the macro-architecture of ECM. Protocol 4 (P4), however, was not efficient in removing all the cells from the matrix. P2, where CHAPS buffer and SDS were employed, ${ }^{15}$ succeeded in the removal of nucleic material, nevertheless failed in the cost-effectiveness analysis when compared to the other studied methods. We also compared a complementary method of P3 where no enzymatic digestion was applied (P3 no Enz) and tested its efficiency in removing the cells. Surprisingly, the P3 no Enz protocol produced acellular scaffolds without any need to add nucleases, as opposed to various other studies on dermis decellularisation ${ }^{12,13}$ where enzymatic digestion plays an important part in the decellularisation process. Moreover, the time frame of scaffolds production was shorter $(<48 \mathrm{~h})$ when compared to the other protocols ( $\sim$ four days), which is very desirable in regenerative medicine and future commercialisation.

It is recognised that the majority of methods for decellularisation can result in disruption of tissue architecture and potential loss of surface structure and composition, ${ }^{12,14}$ compromising the ability of the scaffold to 

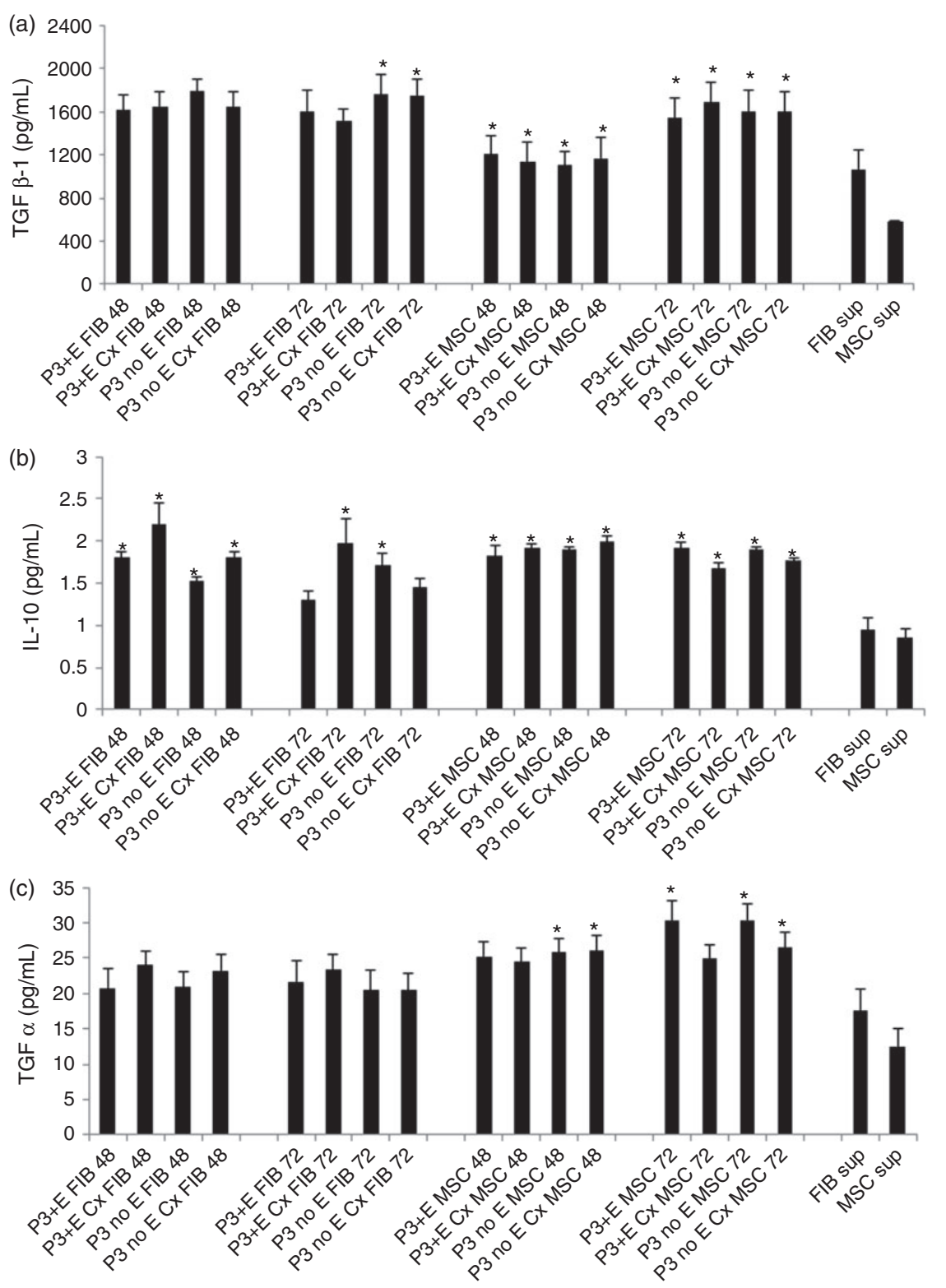

Figure 5. In vitro biocompatibility test: cytokine profile of bone marrow-derived mesenchymal stem cells (MSC) and fibroblasts (FIB). After either MSC or FIB were co-cultured with dermal scaffolds obtained from either protocols P3 or P3 no Enz (crosslinked with genipin $0.5 \%(\mathrm{Cx})$ or non-crosslinked), the supernatants were tested by assessing the release of (a) TGF $\beta$-I, (b) IL-I0, and (c) TNF- $\alpha$ in the culture medium by ELISA. Each assay was performed in triplicate and from three distinct experiments. $* P<0.05$ significantly different from the concentration of the respective factor measured in the supernatant of cells cultured on their own (FIB sup or MSC sup).

provide mechanical support during the remodelling process. ${ }^{16}$ We therefore tested a simplified method in protocols P3 and P3 no Enz, which was designed to provoke an osmotic shock by using a combination of hypertonic and hypotonic solutions. The main reagents used in this protocol were Tris-HCL, sodium chloride, and disodium EDTA. It has been shown that these compounds, and consequent changes on the $\mathrm{pH}$, can cause swelling and shrinkage on the components of ECM leading to loss of proteoglycans (PGs).
However, EDTA, a chelating agent that aids in cell dissociation from ECM proteins by sequestering metal ions, is also known to inhibit metalloproteases thereby preventing the degradation of PGs. $^{17}$ Moreover, hydrogen ions from $\mathrm{Tris}-\mathrm{HCl}$ bind to the PG further inhibiting them from swelling, and the lack of PG hydration can inhibit degradation of PGs by proteases. ${ }^{18}$ Methods using enzymatic digestion are expected to impair the ultrastructure of ECM and usually damage fine fibres of the matrix. ${ }^{19}$ This expectation 
was validated on histological examination of P3 no Enz scaffolds, which displayed a preserved dermal architecture of the collagen and elastic fibres comparable to control. Collagen fibres when viewed under the polarised light appeared in red-orange colour, which corresponds to the coarse and thick collagen bundles of the reticular layer of a native dermis. ${ }^{20}$ Dermal elastic fibres are believed to have an important role in providing elastic stretch and recoil to the skin. ${ }^{21}$ Some studies show that exposure to high concentrations of enzymes (including DNase and RNase) causes disruption to the elastic fibres and consequently on the $\mathrm{ECM},{ }^{22}$ which corroborates with the results we found after P1 and P3. Although some authors have reported that prolonged exposure to enzymes combined with EDTA causes disruption to the ECM, affecting the structure of collagen fibrils, ${ }^{23}$ our results showed no significant damage to the ECM components in decellularised dermis using osmotic shock and nucleases (P3). Moreover, P3 was the only protocol, which was successful in removing cells from the epidermis when tested in full-thickness skin (Supplementary Figure 2), probably because the epidermis is more cellular and needs the combination of the osmotic shock and nucleases. We also tested an extended duration of trypsin digestion on dermis (extensive data not shown) for comparison and analysis under polarised light. Images showed that under polarised light PSR-stained fibres appear yellow in colour with several black gaps, indicating that the collagen fibres in the dermis could be damaged after long enzymatic incubation (Supplementary Figure 1). Some authors have also used trypsin as a decellularising agent but for shorter period of time and shown it to be suitable for decellularising dermis and epidermis of full-thickness skin samples. ${ }^{13}$ Nonetheless, the results from this study are in agreement with other studies showing that different decellularisation methods may succeed differently depending on tissue type, source, and intended clinical application. ${ }^{24}$

In our $\mathrm{P} 1$ protocol, the main reagents were Triton-X and SOC. Triton is a non-ionic detergent that has the function of removing cell residues and SOC is an ionic detergent that is especially useful for disrupting and dissociating protein interactions. ${ }^{14}$ Although some authors have shown that Triton-X did not have a noticeable effect on the GAG content of the porcine dermis samples, ${ }^{12}$ it has been shown that Triton-X especially when combined with other detergents or enzymatic digestion can lead to the loss of major GAG content, resulting in early tissue degradation. ${ }^{22}$ Additionally, other studies have shown that dermis decellularised using Triton-X showed a tendency in retaining lesser collagen when compared to control dermis. ${ }^{2,25}$ These studies can be correlated with our results to some extent as we demonstrated a tendency to decrease collagen and GAGs and no apparent loss in elastic fibres in the matrix after decellularisation using P1.

\section{Ultrastructural assessment and mechanical properties of scaffolds}

In addition to the effects that decellularisation steps may have upon on the properties of ECM scaffolds, ${ }^{12}$ it has been shown that collagen crosslinking can markedly change the surface topography of the ECM. ${ }^{26}$ Exogenous crosslinking agents, such as glutaraldehyde, ribose, formaldehyde, ethyl-(dimethyl aminopropyl) carbodiimide, and genipin have been used to enhance biomechanical properties of collagen-based biomaterial. ${ }^{27,28}$ Amongst them, genipin seems to confer comparatively favourable mechanical properties and cell compatibility to scaffolds. ${ }^{8,28}$

In our study, SEM examinations showed that genipin-crosslinked dermal scaffolds, as expected, had a more compact structure when compared to the noncrosslinked samples. In fact, depending on the particular application, it may be advantageous to produce dense crosslinked scaffolds to limit the degree of subsequent scaffold degradation, which is especially crucial when scaffolds are intended to be used as permanent and non-absorbable scaffolds. SEM analysis also showed that the samples which underwent enzymatic digestion (P1 and $\mathrm{P} 3$ ) presented a loosened collagen meshwork when compared to control samples. Controversially, P3 no Enz samples demonstrated preserved collagen bundles comparable to non-decellularised controls. The differences in the dermal microarchitecture observed in dermal scaffolds may be in part caused by a disruption in the interaction of PG and collagen, ${ }^{29}$ which in turn may be due to the loss in the PG observed in $\mathrm{P} 1 \quad(>40 \%)$ and $\mathrm{P} 3 \quad(\sim 34 \%)$. Another explanation could be the loss of elastin fibres after exposure to enzymes causing a disruption on the organisation of the tissue. ${ }^{22}$ Although SEM analysis showed some differences in the collagen meshwork disposition between samples P1 and P3, the ultra-structural pattern of collagen fibrils, observed by TEM, remained similar to control. TEM images revealed sequential arrangement of fibrils with black and white bands at a bandwidth of approximately $65 \mathrm{~nm}$, which is the typical pattern of collagen fibrils under TEM examination. ${ }^{30}$ Altogether these results seem to reveal that modification of collagen fibrils or loss of ultra-structural orientation might be minimal or altered to a small extent mainly in the P3 no Enz scaffolds. These data are consistent with the analysis of SDS-PAGE for collagen type I in our dermal scaffolds. Images of the electrophoresis gel showed that the known helical structure of type I collagen was displayed in all samples 
comparable to the control, and relevantly P3 no Enz protocol retained collagen in greatest abundance. These results are corroborated by Chen et al., ${ }^{13}$ showing that decellularisation processes using enzymes produced a looser meshwork on SEM images, yet type I collagen polypeptide chains seen in the SDS-PAGE were preserved amongst different decellularisation processes.

Nevertheless, some significant differences in the biomechanical tests were observed, indicating that mechanical properties can be affected by different decellularisation methods, resulting in changes on the YM values. $\mathrm{P} 1$ and $\mathrm{P} 3$ showed a decrease in the tensile strength of scaffolds, whereas P3 no Enz samples displayed on average YM values similar to control dermis. Exogenous crosslinking introduces a stiffening effect in the dermal scaffolds, enhancing its mechanical properties as discussed in the literature. ${ }^{8}$ Nevertheless, our results showed that genipin-crosslinking showed a tendency in increasing YM values but did not significantly alter the biomechanical properties of dermal scaffolds. This finding is in line with other studies showing no significant alteration in the mechanical properties after crosslinking with genipin. ${ }^{27,31}$

\section{Biocompatibility of dermal scaffolds and trophic factors analysis}

ECM not only acts as a physical framework, but also exerts a profound effect on cell shape and behaviour, including cell adhesion, spreading, migration, proliferation, and differentiation. ${ }^{32}$ Ability of porcine dermis scaffolds to support in vitro cell growth was tested using an in vitro Boyden-type chemotaxis assay.

Migration and adhesion is required for cells to interact with scaffolds, and it is initiated by a variety of cell surface adhesion proteins and receptors. Previous studies have shown that products of ECM scaffolds can recruit a population of progenitor cells both in vitro and in vivo. ${ }^{6,11,13,33}$ In vivo processes of skin repair and remodelling are orchestrated by distinct cell populations; among them mesenchymal stem cells (MSC) and fibroblasts are known to exert an important role. ${ }^{34}$ Our results showed that the two decellularisation protocols (P3 and P3 no Enz) did not differ to a great extent in their effects on signalling BM-MSC to migrate and adhere to the scaffolds. However, when we tested the capacity of fibroblasts seeded on the scaffolds to adhere and proliferate, non-crosslinked scaffolds proved to be more favourable for proliferation when compared to the crosslinked ones after $72 \mathrm{~h}$. This is aligned with the premise that the collagen fibre architecture of ECM scaffolds not only plays a critical role in determining its biomechanical behaviour, ${ }^{24}$ but also contributes to the regulation of cellular functions of the tissue. ${ }^{7}$ A non-crosslinked matrix, with rougher surfaces, usually provides the ideal topography for cell attachment. ${ }^{35}$ This may facilitate migration and proliferation of cells, and eventually promote the revascularisation and dermal regeneration of implanted scaffolds, which may be beneficial when the final clinical application is for tissue remodelling.

While the original hypothesis underlying stem cell regenerative therapies was focused on functional recovery as a consequence of MSC differentiation, ${ }^{36}$ it is now clear that other mechanisms of action are at play. A recent paradigm shift has emerged suggesting that biomolecules synthesised by MSC may be as important, if not more so, than differentiation of the cells in eliciting functional tissue repair. ${ }^{37} \mathrm{We}$ tested the capacity of cells (BM-MSC and fibroblasts) to release trophic factors when co-cultured with decellularised dermis (P3 and P3 no Enz) and observed some significant changes when compared to the supernatant of the cells on its own. Transforming growth factor-beta 1 (TGF- $\beta 1$ ) released by BM-MSC increased in response to co-culture in both crosslinked and non-crosslinked scaffolds from both protocols. This can be advantageous as it has been shown that TGF- $\beta 1$ is one of the key factors of immunomodulation by MSC. ${ }^{38,39}$ it stimulates these cells by autocrine and paracrine effects to proliferate and also to produce ECM components. ${ }^{40}$ MSCs synthesise and release TGF- $\beta 1$ that not only increases $\alpha 1$-procollagen production ${ }^{41}$ but have also been demonstrated to increase other ECM proteins, such as hyaluronan when in contact with scaffolds. ${ }^{42}$ Surprisingly, an up-regulation of TNF- $\alpha$ was also observed when BM-MSCs were co-cultured with P3 no Enz decellularised scaffolds after $48 \mathrm{~h}$. TNF- $\alpha$ is a potent pro-inflammatory cytokine that plays an important role not only in immunity and inflammation but also in the control of cell proliferation, differentiation, and apoptosis. ${ }^{43}$ Although TNF- $\alpha$ may be critical as pro-inflammatory molecule, we underpin its proliferative function, which is explored in the literature showing that MSCs stimulate tissue regeneration and accelerate cutaneous wound repair by direct differentiation of MSCs to tissue cells or secretion of paracrine factors, including TNF- $\alpha{ }^{44}$ Therefore, upregulation of TNF- $\alpha$ by BM-MSC may also demonstrate involvement in differentiation and proliferation, which is essential for tissue regeneration.

Interleukin (IL)-10 levels were also enhanced by both cell types. The immuno-regulatory cytokine IL-10 plays an important role in preventing excessive inflammation and tissue damage ${ }^{45}$ hence it can be also favourable in this scenario. In addition to these abovementioned trophic factors, there are also other cytokines and growth factors, which are known to play a very important role in tissue remodelling. Therefore, further investigations are being carried out for the 
validation of active molecules in our scaffolds (manuscript in preparation).

These findings show that BM-MSC and fibroblast seeded dermal scaffolds are a pliable material with potential dual function as a mechanical reconstructive agent and as a biolayer of cells for targeted delivery and remodelling. Nonetheless, certain caveats exist in interpreting this study, as the in vitro assays used here are unlikely to fully mimic conditions during clinical use of the materials; however, they do reflect many of the basic processes thought to be important for tissue regeneration. Additionally, the cytotoxicity assessment of the scaffolds using a novel approach to the BoydenType in vitro assay has significant advantages such as limiting the use of animals as well as quantitatively measuring the biological properties that would otherwise be difficult to assess in vivo.

\section{Conclusion}

Five decellularisation methods were tested and compared in order to obtain porcine dermal scaffolds suitable for biomedical applications. The osmotic shock method applied (both with and without nucleases) effectively removed cellular material including DNA and debris. Histological, SEM and TEM examinations, as well as analysis by SDS-PAGE revealed that these scaffolds retained structural properties comparable to a native dermal matrix. Under optimum conditions and using a non-enzymatic decellularisation method (P3 no Enz) however, dermal scaffolds were produced at lower cost and under two days. Other components of ECM such as GAGs and elastin fibres were also preserved. The in vitro biocompatibility tests showed that these scaffolds not only supported cell migration, adhesion, and proliferation, but also stimulated the cells to release trophic factors essential for tissue regeneration.

\section{Acknowledgements}

The authors thank Kajal Sachdev and Farhana Surti for their valuable contribution on the histological analysis; and Kallyne O. Mimura for her expert assistance with the ELISA assay.

\section{Declaration of conflicting interests}

None declared.

\section{Funding}

This research received no specific grant from any funding agency in the public, commercial, or not-for-profit sectors.

\section{References}

1. Kirsner RS. The use of Apligraf in acute wounds. J Dermatol 1998; 25: 805-811.
2. Horch RE, Kopp J, Kneser U, et al. Tissue engineering of cultured skin substitutes. J Cell Mol Med 2005; 9: 592-608.

3. Shevchenko RV, James SL and James SE. A review of tissue-engineered skin bioconstructs available for skin reconstruction. $J$ R Soc Interface 2010; 7: 229-258.

4. Gentile P, Colicchia GM, Nicoli F, et al. Complex abdominal wall repair using a porcine dermal matrix. Surg Innov 2013; 20: NP12-15.

5. Supp DM and Boyce ST. Engineered skin substitutes: practices and potencials. Clin Dermatol 2005; 23: 403-412.

6. Agrawal V, Tottey S, Johnson SA, et al. Recruitment of progenitor cells by an extracellular matrix cryptic peptide in a mouse model of digit amputation. Tissue Eng Part A 2011; 17: 2435-2443.

7. Sundararaghavan $\mathrm{HG}$, Monteiro GA, Lapin NA, et al. Genipin-induced changes in collagen gels: correlation of mechanical properties to fluorescence. J Biomed Mater Res A 2008; 87: 308-320.

8. Qiu J, Li J, Wang G, et al. In vitro investigation on the biodegradability and biocompatibility of genipin crosslinked porcine acellular dermal matrix with intrinsic fluorescence. ACS Appl Mater Interfaces 2013; 5: 344-350.

9. Wolf MT, Daly KA, Reing JE, et al. Biologic scaffold composed of skeletal muscle extracellular matrix. Biomaterials 2012; 33: 2916-2925.

10. Brown AL, Brook-Allred TT, Waddell JE, et al. Bladder acellular matrix as a substrate for studying in vitro bladder smooth muscle-urothelial cell interactions. Biomaterials 2005; 26: 529-543.

11. Kulig KM, Luo X, Finkelstein EB, et al. Biologic properties of surgical scaffold materials derived from dermal ECM. Biomaterials 2013; 34: 5776-5784.

12. Reing JE, Brown BN, Daly KA, et al. The effects of processing methods upon mechanical and biologic properties of porcine dermal extracellular matrix scaffolds. Biomaterials 2010; 31: 8626-8833.

13. Chen R-N, Ho H-O, Tsai Y-T, et al. Process development of an acellular dermal matrix (ADM) for biomedical applications. Biomaterials 2004; 25: 2679-2686.

14. Crapo PM, Gilbert TW and Badylak SF. An overview of tissue and whole organ decellularization processes. Biomaterials 2011; 32: 3233-3243.

15. Gui L, Muto A, Chan SA, et al. Development of decellularized human umbilical arteries as small-diameter vascular grafts. Tissue Eng Part A 2009; 15: 2665-2676.

16. Haag J, Baiguera S, Jungebluth $\mathrm{P}$, et al. Biomechanical and angiogenic properties of tissue-engineered rat trachea using genipin cross-linked decellularized tissue. Biomaterials 2012; 33: 780-789.

17. Campo RD and Betz RR. Loss of proteoglycans during decalcification of fresh metaphyses with disodium ethylenediaminetetraacetate (EDTA). Calcif Tissue Int 1987; 41: 52-55.

18. Campo RD. Effects of cations on cartilage structure: swelling of growth plate and degradation of proteoglycans induced by chelators of divalent cations. Calcif Tissue Int 1988; 43: 108-121. (Erratum in: Calcif Tissue Int 1989 Mar; 44: 234). 
19. Gillies AR, Smith LR, Lieber RL, et al. Method for decellularizing skeletal muscle without detergents or proteolytic enzymes. Tissue Eng Part C Methods 2011; 17: 383-389.

20. Rich L and Whittaker P. Collagen and Picrosirius red staining: a polarized light assessment of fibrilar hue and special distribution. J Morphol Sci 2005; 22: 97-104.

21. Starcher B, Aycock RL and Hill CH. Multiple roles for elastic fibers in the skin. J Histochem Cytochem 2005; 53 : 431-443.

22. Liao J, Joyce EM and Sacks MS. Effects of decellularization on the mechanical and structural properties of the porcine aortic valve leaflet. Biomaterials 2008; 29: 1065-1074.

23. Gilbert TW, Sellaro TL and Badylak SF. Decellularization of tissues and organs. Biomaterials 2006; 27: 3675-3683.

24. Badylak SF, Freytes DO and Gilbert TW. Extracellular matrix as a biological scaffold material: structure and function. Acta Biomater 2009; 5: 1-13.

25. Rosso F, Giordano A, Barbarisi M, et al. From cell-ECM interactions to tissue engineering. J Cell Physiol 2004; 199: 174-180.

26. Brown BN, Barnes CA, Kasick RT, et al. Surface characterization of extracellular matrix scaffolds. Biomaterials 2010; 31: 428-437.

27. Elder BD, Moran A and Athanasiou KA. Beneficial effects of exogenous crosslinking agents on selfassembled tissue engineered cartilage construct biomechanical properties. J Mech Med Biol 2011; 11: 433-443.

28. Madhavan K, Belchenko D and Tan W. Roles of genipin crosslinking and biomolecule conditioning in collagenbased biopolymer: potential for vascular media regeneration. J Biomed Mater Res A 2011; 97A: 16-26.

29. Meigel WN, Gay S and Weber L. Dermal architecture and collagen type distribution. Arch Derm Res 1977; 259: $1-10$.

30. Zuo H, Peng D, Zheng B, et al. Regeneration of mature dermis by transplanted particulate acellular dermal matrix in a rat model of skin defect wound. J Mater Sci Mater Med 2012; 23: 2933-2944.

31. Yerramalli CS, Chou AI, Miller GJ, et al. The effect of nucleus pulposus crosslinking and glycosaminoglycan degradation on disc mechanical function. Biomech Model Mechanobiol 2007; 6: 13-20.

32. Ingber $\mathrm{SN}$ and Folkman SN. How does extracellular matrix control capillary morphogenesis? Cell 1989; 58: 803-805.

33. Raghavan SS, Woon CY, Kraus A, et al. Optimization of human tendon tissue engineering: synergistic effects of growth factors for use in tendon scaffold repopulation. Plast Reconstr Surg 2012; 129: 479-489.

34. Marcopoulou CE, Vavouraki HN, Dereka XE, et al. Proliferative effect of growth factors TGF-beta1, PDGF-BB and rhBMP-2 on human gingival fibroblasts and periodontal ligament cells. J Int Acad Periodontol 2003; 5: 63-70.

35. Francis L, Meng D, Knowles JC, et al. Multi-functional $\mathrm{P}(3 \mathrm{HB})$ microsphere/45S5 Bioglass-based composite scaffolds for bone tissue engineering. Acta Biomater 2010; 6: 2773-2786.

36. Williams AR and Hare JM. Mesenchymal stem cells: biology, pathophysiology, translational findings, and therapeutic implications for cardiac disease. Circ Res 2011; 109: 923-940.

37. Baraniak PR and McDevitt TC. Stem cell paracrine actions and tissue regeneration. Regen Med 2010; 5: 121-143.

38. Di Nicola M, Carlo-Stella C, Magni M, et al. Human bone marrow stromal cells suppress T-lymphocyte proliferation induced by cellular or nonspecific mitogenic stimuli. Blood 2002; 99: 3838-3843.

39. Patel SA, Meyer JR, Greco SJ, et al. Mesenchymal stem cells protect breast cancer cells through regulatory T cells: role of mesenchymal stem cell-derived TGF-beta. J Immunol 2010; 184: 5885-5894.

40. Leask A and Abraham DJ. TGF- $\beta$ signaling and the fibrotic response. FASEB J 2004; 18: 816-827.

41. Kjaer M. Role of extracellular matrix in adaptation of tendon and skeletal muscle to mechanical loading. Physiol Rev 2004; 84: 649-680.

42. Johnson BQ, Fox R, Chen X, et al. Tissue regeneration of the vocal fold using bone marrow mesenchymal stem cells and synthetic extracellular matrix injections in rats. Laryngoscope 2010; 120: 537-545.

43. Schling P, Rudolph C, Heimerl S, et al. Expression of tumor necrosis factor alpha and its receptors during cellular differentiation. Cytokine 2006; 33: 239-245.

44. Heo SC, Jeon ES, Lee IH, et al. Tumor necrosis factor-aactivated human adipose tissue-derived mesenchymal stem cells accelerate cutaneous wound healing through paracrine mechanisms. J Invest Dermatol 2011; 131: 1559-1567.

45. Payne NL, Sun G, McDonald C, et al. Human adiposederived mesenchymal stem cells engineered to secrete IL10 inhibit APC function and limit CNS autoimmunity. Brain Behav Immun 2013; 30: 103-114. 